\title{
Understanding the Basic Concepts of Liver Transplantation
}

\section{Chandan Kumar Kedarisetty ${ }^{*}$, Senthil Kumar ${ }^{2}$, Vanarasi Y Bhargav $^{1}$, Mu Karthikeyan', Tamarai Selvan' ${ }^{1}$, Babu Elangovan², Jayanthi Venkataraman ${ }^{1}$}

${ }^{1}$ Departments of Hepatology, Sri Ramachandra Institute of Higher

Education and Research, Porur, Chennai, India

${ }^{2}$ Hepatobiliary Surgery and Liver Transplantation, Sri Ramachandra

Institute of Higher Education and Research, Porur, Chennai, India

*Corresponding Author: Chandan Kumar Kedarisetty, Department of

Hepatology, Sri Ramachandra Institute of Higher Education and Research,

Porur, Chennai, India.

DOI: $10.31080 /$ ASGIS.2020.03.0115
Received: January 04, 2020

Published: January 29, 2020

(C) All rights are reserved by Chandan Kumar

Kedarisetty., et al.

\begin{abstract}
Most of the patients referred to a specialized hepatology center suffer from decompensation of end-stage chronic liver disease or acute-on-chronic liver failure. A subset of patients with no pre-existing illness can present as acute liver failure. The only recommended treatment of choice is liver transplantation. Hence, it is important to understand the indications for liver transplantation, types of liver transplantation and a general overview of immunosuppression.

Keywords: Hepatology; Liver Transplantation; Liver Failure
\end{abstract}

\section{Abbreviations}

LT: Liver Transplantation; HCC: Hepatocellular Carcinoma; ESLD: End Stage Liver Disease; CTP: Child-Turcotte-Pugh; MELD: Model for End Stage Liver Disease, MELID-Na: Model for End Stage Liver Disease Sodium; UNOS: United Network for Organ Sharing; SBP: Spontaneous Bacterial Peritonitis; HPS: Hepatopulmonary Syndrome; POPH: Porto-Pulmonary Hypertension; mPAP: Mean Pulmonary Artery Pressure; PVR: Pulmonary Vascular Resistance; HE: Hepatic Encephalopathy; OHE: Overt Hepatic Encephalopathy; MHE: Minimal Hepatic Encephalopathy; ALF: Acute Liver Failure; IEI: Icterus to Encephalopathy Interval; ACLF: Acute on Chronic Liver Failure; CLD: Chronic Liver Disease; APASL: Asian Pacific Association for the Study of the Liver; LDLT: Living Donor Liver Transplantation; DDLT: Deceased Donor Liver Transplantation; A2ALL: Adult-to-Adult LDLT; DBD: Donation After Brain Death; DCD: Donation After Cardiac Death; CIT: Cold Ischemia Time; SLT: Split Liver Transplantation; CNIs: Calcineurin Inhibitors.

\section{Introduction}

It has been more than 50 years since Prof Thomas Starzl and colleagues [1] performed the first successful human liver transplantation (LT) in the United States. In the following years, LT gradually established its role as definitive therapy for patients with acute liver failure and end-stage liver disease, and later on for selected patients with hepatocellular carcinoma (HCC). Over this period, rapid advances have taken place in pre-operative management of these critically ill liver diseases patients, sophisticated operative techniques, implementation of optimal post-transplant immunosuppressive regimens to balance between adequate immunosuppression to prevent rejection and not too much to cause infection. For a successful outcome in LT, it needs a multidisciplinary approach with a hepatologist, liver transplant surgeon and liver intensivist who serve as pillars of the program and ably supported by allied specialities like cardiology, pulmonology, nephrology, infectious diseases, transfusion medicine, radiology and so on. Timely transplant is the key to success, failing which patients do end up becoming too sick and are not in a fit state for LT. Hence it is important to understand that referral for liver transplant should be considered at the onset of decompensation and not just based on MELD criteria alone due to long waiting list especially in a cadaveric transplant setting.

It is prudent to identify these patients of end stage liver disease (ESLD) early, triage patients needing LT, optimize them medically prior to transplant, establish standard working protocols for posttransplant and long-term immunosuppression with careful tailoring of immunosuppression, keeping strict vigilance on possible post-transplant metabolic complications at any point of time. The goal of such a systematic and protocolised approach is to maximise good patient and graft outcomes in a replicable manner across the board amongst various centres in the world. 


\section{Common indications for liver transplant}

The indications for liver transplantation may be classified under different headings as summarised in Box 1 . The recipient should be under 75 years of age, with no major cardiovascular, pulmonary or neurological condition that would preclude a major surgery under general anaesthesia.

1. Decompensated liver cirrhosis
a. Ethanol related
b. Viral causes (Hepatitis B or Hepatitis C)
c. Non-alcoholic fatty liver disease
d. Autoimmune hepatitis
e. Chronic cholestatic disorders like PBC/ PSC
f. Secondary biliary cirrhosis
g. Cryptogenic

2. Metabolic liver disease
a. Wilson's disease
b. Alpha-1 antitrypsin deficiency
c. Familial amyloid polyneuropathy
d. Tyrosinemia
e. Primary hyperoxaluria

3. Neoplasms
a. Hepatocellular carcinoma
b. Early Cholangiocarcinoma
i. Hilar
ii. Intrahepatic

c. Unresectable neuroendocrine metastases

d. Unresectable colorectal metastases (selected)

e. Unresectable hepatoblastoma

f. Large hepatic hemangioendothelioma

g. Extensive adenomatosis

4. Cystic liver diseases
a. Polycystic liver disease
b. Extensive hepatic hydatid cysts

5. Vascular diseases of liver

a. Chronic hepatic venous outflow obstruction

b. Symptomatic Abernethy malformation

6. Trauma
a. Extensive liver trauma (as last resort)
b. Extensive bilio-vascular injury (as last resort)

7. Liver failure
a. Acute liver failure
b. Acute-on-chronic liver failure
c. Post hepatectomy liver failure
d. Primary non-function post-transplant

8. Miscellaneous
a. Extrahepatic biliary atresia
b. Progressive familial intrahepatic cholestasis

\section{Box 1}

Transplantation for decompensated liver cirrhosis

The natural history of cirrhosis is characterised by an asymptomatic compensated phase followed by a decompensated phase, marked by the development of overt clinical signs, the most frequent of which are ascites, bleeding, encephalopathy, and jaundice [2]. The transition from compensated asymptomatic cirrhosis to decompensated cirrhosis occurs at a rate of about $5 \%$ to $7 \%$ per year [3]. Once decompensation occurs, cirrhosis becomes a systemic disease, with multi-organ/system dysfunction [4]. Basically, after the onset of decompensation, there is immune paresis which increases the risk of a cirrhotic patient to infections, leading to an increase in morbidity and mortality5. Consequently, the median survival drops from 12 years in compensated cirrhotic stage to just 2 years in a decompensated cirrhotic [3].

Next, it is important to understand which patients with liver decompensation should be listed for LT as a curative option. Child and Turcotte [6] described in 1964 the first classification system for the prediction of survival among patients with cirrhosis complicated by variceal bleeding undergoing portosystemic shunt surgery, which was based on three clinical variables namely ascites, hepatic encephalopathy, and nutritional status, in addition to two laboratory variables namely serum bilirubin and albumin. This classification divided patients with cirrhosis into 3 categories, based on their mortality risk for major surgery. In 1973, Pugh and colleagues [7] modified the original Child-Turcotte classification, assigning a score ranging from 1 to 3 to each of the 5 variables and replacing nutritional status by prothrombin time. The modified score was renamed the Child-Turcotte-Pugh (CTP) score. However, these criteria were not effective in listing for LT as three of the five variables were subjective and led to unnecessary listing in an already constrained availability of cadaveric organs.

Subsequently, Mayo clinic came out with Model for End Stage Liver Disease (MELD) scoring system [8]. It was originally called Mayo End-Stage Liver Disease model as a tool to predict short-term survival (3 months) after undergoing transjugular intrahepatic shunt placement [9]. It is calculated using a logarithmic equation comprising of three laboratory variables namely serum creatinine, total bilirubin and INR. Bilirubin and INR reflect the degree of liver dysfunction and inclusion of creatinine reiterates the important prognostic implications of involvement of kidney function in a decompensated cirrhotic [10]. At present, a patient is listed for liver transplantation if MELD score [11] is $\geq 15$. As intended, the presence of complications of end stage cirrhosis, including ascites, hepatic encephalopathy, variceal bleeding, and spontaneous bacterial peritonitis, did not affect the predictive value of the MELD score.

MELD score was superior to earlier used CTP score because it was based on only laboratory parameters, which are measured in a continuous scale, easily available and reproducible. Most importantly, its main strength was in its validity as a mathematical model 
to predict mortality in patients with cirrhosis. The MELD score became the best option available for the determination of disease severity in order to allocate donor organs to the sickest patient first and not based on geography or local preferences or duration of waiting on the list and other subjective parameters $[12,13]$.

\section{Addition of serum sodium to MELD score}

Dilutional hyponatremia is a common phenomenon in the pathophysiology of portal hypertension. Due to reduced arterial blood volume, there is raised antidiuretic hormone levels, which results in reduced free water excretion in the collecting tubules of the kidneys, leading to increased free water content in blood and consequently hyponatremia [4]. In an elegant population-based study, the authors had found $21.6 \%$ cirrhotics with serum sodium $\leq 130 \mathrm{meq} / \mathrm{dl}$ and $5.7 \%$ were $\leq 125 \mathrm{meq} / \mathrm{dl}$ [14]. In another retrospective study, reduction of serum sodium from $135 \mathrm{meq} / \mathrm{dl}$ by every $1 \mathrm{meq} / \mathrm{dl}$, reduced 3-month survival by $12 \%$ [15]. Addition of sodium to MELD score improved the accuracy of mortality prediction in those with lower MELD scores at the time of listing. The revised MELD sodium formula (MELD Na) incorporated serum sodium value into MELD score in those patients with serum sodium $\leq 135 \mathrm{meq} / \mathrm{dl}$ and $\geq 120 \mathrm{meq} / \mathrm{dl}$ [16]. Subsequently United Network for Organ Sharing (UNOS) in US adopted the use of MELD Na score for patient listing for liver transplantation with an initial MELD greater than 11 and serum sodium $<137 \mathrm{meq} / \mathrm{dL}$ and $\geq 125$ meq/dL. Those with $<125 \mathrm{meq} / \mathrm{dl}$ did not get any additional MELD increase. By adopting MELD Na score, a study on UNOS database showed 7\% reduction in wait list mortality [17].

\section{Specific conditions with MELD exception criteria}

Patients suffering from selected conditions as a sequelae of ESLD can be considered for LT despite the MELD score being < 15. These criteria have been shown to be independently associated with morbidity and mortality in a cirrhotic and hence have been adopted by UNOS as a policy decision to give more weightage to those patients who are listed for liver transplant on cadaveric waiting list. However, the same doesn't hold true in many countries like India where organ donation is very scarce and hence those patients with any of these criteria are mostly counselled for living donor liver transplant option as the best viable option.

\section{Hepatopulmonary syndrome}

The diagnostic triad for hepatopulmonary syndrome (HPS) is presence of portal hypertension, hypoxemia with $\mathrm{PaO}_{2}<80 \mathrm{mmHg}$ or alveolar - arterial oxygen gradient $\geq 15 \mathrm{~mm} \mathrm{Hg}$ at room air $(\geq \mathrm{Pa}$ tients suffering from selected conditions as a sequelae of ESLD can be considered for LT despite the MELD score being $<15$. These criteria have been shown to be independently associated with morbidity and mortality in a cirrhotic and hence have been adopted by UNOS as a policy decision to give more weightage to those patients who are listed for liver transplant on cadaveric waiting list. However, the same doesn't hold true in many countries like India where organ donation is very scarce and hence those patients with any of these criteria are mostly counselled for living donor liver transplant option as the best viable option $20 \mathrm{mmHg}$ for individuals more than 65 years of age) in absence of an underlying intrinsic lung disease [18]. HPS is a consequence of intrapulmonary vascular shunts that bypass the laminar blood flow and impair the oxygenation in the lungs leading to progressive hypoxemia. These patients get a MELD score of 22 (equivalent to 15\% 3-month mortality) assigned during listing, with increase in MELD points equivalent to $10 \%$ increase in mortality every 3 months, provided $\mathrm{PaO} 2$ remains less than $60 \mathrm{~mm} \mathrm{Hg}$.

\section{Porto-pulmonary hypertension}

A diagnosis of porto-pulmonary hypertension ( $\mathrm{PoPH}$ ) is considered in a patient with established portal hypertension in the absence of other causes of pulmonary artery or venous hypertension such as chronic thromboembolism, chronic lung disease/hypoxia; chronic left heart disease [19]. For POPH, the basic requirement is the documentation of initial mean pulmonary artery pressure (mPAP) $\geq 35 \mathrm{~mm} \mathrm{Hg}$, transpulmonary gradient of $12 \mathrm{~mm} \mathrm{Hg}$, and pulmonary vascular resistance (PVR) $>400$ dynes $/ \mathrm{cm}^{-5}$, that are consistent with at least moderate pulmonary hypertension. These patients get a MELD score of 22 assigned at the time of listing, with an increase in MELD points by 2 points (equivalent to $10 \%$ increase in mortality) every 3 months, provided $\mathrm{PaO}_{2}$ remains less than $60 \mathrm{~mm} \mathrm{Hg}$.

\section{Hepatocellular carcinoma}

The entire treatment perspective of HCC changed with the landmark publication by Mazzaferro and colleagues [20] wherein they reported a curative potential of LT with a one-year survival of $85 \%$ and a five-year survival of more than $70 \%$ and recurrence rate of less than $10 \%$ in cirrhotics with HCC within Milan criteria (either a single lesion $\leq 5 \mathrm{~cm}$ in maximum diameter or up to 3 lesions with a maximum diameter of any lesion of $3 \mathrm{~cm}$ ). These stringent criteria were subsequently expanded by Yao and colleagues 21 to UCSF criteria i.e. a single tumor with size $\leq 6.5 \mathrm{~cm}$ or maximum of 3 lesions up to $4.5 \mathrm{~cm}$ and cumulative tumor burden $\leq 8 \mathrm{~cm}$ ) with similar survival benefit. In 2003, UNOS [22] allotted MELD exception score of 22 for stage 2 tumors (lesions within Milan criteria) with capping MELD score maximum up to 34. Recently HALT-HCC score [23], based on tumour burden, alpha fetoprotein and MELD sodium has been shown to predict mortality in patients waiting for liver transplant with HCC, with a hazard ratio of 1.06 (95\% CI 1.05 - 1.07).

\section{Hepatic encephalopathy}

Hepatic encephalopathy (HE) reflects a spectrum of neuropsychiatric abnormalities seen in patients with liver dysfunction, after exclusion of other known brain pathology. HE is divided into two primary components: overt HE (OHE) and minimal HE (MHE). OHE can be diagnosed clinically, whereas MHE is a subclinical entity manifesting as subtle cognitive dysfunction detected only by a dedicated battery of tests. It has been reported that OHE is present 
in $30-45 \%$ cirrhotics more in advanced liver disease [24]. Whereas, MHE is reported in $60-80 \%$ cirrhotics. Liver transplantation remains the only treatment option for HE that does not improve on any other treatment.

Rare conditions [25] include patients with a diagnosis of familial amyloid polyneuropathy, cystic fibrosis, primary hyperoxaluria.

\section{Acute liver failure}

Acute liver failure (ALF) is as a clinical syndrome entity wherein a patient with no pre-existing liver disease, develops jaundice followed by encephalopathy within 4 weeks after an acute insult [26]. Of the many classifications, the most commonly followed 0' Grady system [27] is based on icterus to encephalopathy interval (IEI). ALF is classified as hyperacute if IEI is less than a week, acute if it is between $1-4$ weeks and subacute if it between $4-12$ weeks. Transplant free survival is relatively better with hyperacute ALF compared to acute and worst with subacute. The only choice of treatment in such situations is LT as a long-term definitive option. Most of the centres including our centre use Kings college criteria [28] to identify patients of ALF for emergency listing for LT.

\section{Acute liver failure}

Acute liver failure (ALF) is as a clinical syndrome entity wherein a patient with no pre-existing liver disease, develops jaundice followed by encephalopathy within 4 weeks after an acute insult [26]. Of the many classifications, the most commonly followed O' Grady system [27] is based on icterus to encephalopathy interval (IEI). ALF is classified as hyperacute if IEI is less than a week, acute if it is between $1-4$ weeks and subacute if it between $4-12$ weeks. Transplant free survival is relatively better with hyperacute ALF compared to acute and worst with subacute. The only choice of treatment in such situations is LT as a long-term definitive option. Most of the centres including our centre use Kings college criteria [28] to identify patients of ALF for emergency listing for LT.

\section{Acute - on - chronic liver failure (ACLF)}

This is a distinct entity in the spectrum of chronic liver disease (CLD), that is characterised by a rapid downhill clinical course and a poor outcome. ACLF was initially defined by Sen., et al. [29], as the development of recent onset ( $<3$ months) of ascites, jaundice, hepatic encephalopathy, hepatorenal syndrome, upper gastro-intestinal bleeding in patients with diagnosed or undiagnosed CLD due to the influence of precipitating events such as complications of sepsis, ischemia or additional superimposed liver injury due to alcohol, hepatotropic virus and hepatotoxic drugs.

The Asian Pacific Association for the Study of the Liver (APASL) consensus [30] subsequently defined ACLF as an acute hepatic insult manifesting as jaundice (serum bilirubin $\geq 5 \mathrm{mg} / \mathrm{dL}$ ) and coagulopathy (INR $\geq 1.5$ ) complicated within 4 weeks by ascites and/or encephalopathy in a patient with previously diagnosed or undiag- nosed CLD. The seminal work from the EASL-CLIF consortium [31] defined ACLF as an acute deterioration of pre-existing CLD, usually related to a precipitating event and associated with increased mortality at 3 months due to multiple organ failures. The potential of reversibility despite high mortality without LT remains the utmost reason for classifying these patients as a distinct group. The probability of reversibility of ACLF is less likely once extra-hepatic organ failure(s) sets in. Even the suitability for LT decreases in the presence of extra-hepatic organ failure. It is therefore advisable to identify the short interim period between the onset of liver failure and before the onset of extra-hepatic organ failure i.e. the potential therapeutic 'golden window'. The recently published AARC score [32] is a good dynamic prognostic index to identify patients early and list them for LT.

\section{Common diseases in pediatric population}

The most indication for liver transplantation in children is extra hepatic biliary atresia [33] with a failed Kasai operation, presenting as decompensated biliary cirrhosis with failure to thrive. Metabolic liver diseases [34] such as galactosemia, tyrosinemia are another important group which need to be identified early to consider timely liver transplantation.

\section{Types of liver transplantation}

Living donor liver transplantation

Liver transplantation is the treatment of choice for select patients with end stage liver disease. However, many patients are unable to receive a transplant because of the shortage of deceased donor livers. In living donor liver transplantation (LDLT), the live donor gives a portion of liver which is transplanted in the patient (recipient). The primary benefit of LDLT is an expedited, well timed and elective transplant that can be performed before the candidate deteriorates to the point that the outcome of the surgery is jeopardized. Consequently, patients that are likely to reap the greatest benefit from LDLT are those with a significant risk for death or deterioration before the anticipated deceased donor liver transplantation (DDLT). There may not be any apparent benefit of LDLT for patients with severe decompensation, a high MELD score, and a long wait time in DDLT list. A landmark Adult-to-Adult LDLT (A2ALL) [35] study sponsored by the National Institutes of Health US, reported that compared to those listed in DDLT list, patients undergoing LDLT had a nearly two-thirds reduction in mortality with an adjusted mortality hazard ratio of $0.35(\mathrm{p}<0.05)$. Most important part of LDLT is donor safety with minimal morbidity should be ensured with a detailed pre-transplant evaluation both technically and medically.

\section{Deceased donor liver transplantation}

Cadaveric LT, that is donation after brain death policy was first proposed at a 1965 CIBA Foundation meeting following report of a successful kidney transplant from a brain-dead donor [36] and subsequently endorsed with formal diagnostic criteria by Harvard 
Medical School in 1968 [37]. The core criteria include complete apnea, brainstem areflexia, and cerebral unresponsiveness, taken together with documentation of an irreversible and permanent loss of central nervous system function. Due to shortage of organs, donor risk index [38] was proposed to determine the donor organ - recipient suitability, so that if a recipient was found to be unfit for a particular donor organ, then another suitable recipient could be selected using this index. Similarly, donor selection criteria have been further expanded [39] to accommodate more recipients for the utilization of the limited donor pool such as steatotic grafts, anti $\mathrm{HBc}$ (total) positive donors, older donors, donors infected with bacterial or fungal infections and so on.

There are broadly 2 types of deceased donor organ donation (procurement; harvesting; retrieval) namely Donation after brain death (DBD) and Donation after cardiac death (DCD). Both need the explicit consent of the family of the deceased person. In DBD, after declaration of brain death, abdominal exploration is performed while the circulation is still being maintained by the beating heart.

An initial dissection to isolate key vascular structures and cannulation of aorta and the portal venous system (via SMV or IMV) is performed in the warm phase. Then, an in situ cold flush is performed through these cannulas after cross clamping the aorta above the celiac vessels, to rapidly cool the liver in what is termed the cold phase. The liver is then resected with its vasculature and bile duct. Cold preservation is then continued till it is ready to be implanted in the recipient. The preservation phase may be static preservation in an ice box or may be dynamic preservation by continuous machine perfusion. The commonly used specialized preservation solutions are the University of Wisconsin (UW), Hystidine-keto-glutarate (HTK or IGL-1 solutions. The time between the start of cold in situ perfusion (Aortic cross clamp) to the time the liver is taken out of ice is called the cold ischemia time (CIT). This should ideally be kept to less than 8 hours, although livers have been successfully transplanted even after 15 hours of CIT.

In DCD, by definition, the heart has already arrested before the procurement starts. The organs inevitably suffer an element of 'warm ischemia' wherein the organs are still warm but deprived of oxygen as there is cessation of circulation. A super rapid technique of laparotomy, cannulation and rapid flush is performed before the liver is dissected out. There is no warm phase in the dissection during this type of operation. The outcomes of DCD are inferior to the DBD livers.

\section{Split liver transplantation}

Split-liver transplantation (SLT) is an advanced surgical technique that allows creation of two functional allografts from one deceased donor liver, conceived by Bismuth and Houssin [40] in 1984. By SLT, the scarcity of deceased donor organs is addressed and commonly, one deceased donor liver can be split between an adult (usually an extended right lobe graft consisting of seg IV to
VIII) and a pediatric recipient (who gets the left lateral segment). True left and right lobe splits can also be performed.

\section{Domino or sequential transplantation}

Herein, a portion of liver allograft from a previous LT recipient is used as a live donor graft for another recipient [41]. This is considered in special situations such as familial amyloid polyneuropathy when there is perennial shortage of organs. Familial amyloid polyneuropathy [42], is an autosomal dominant disease involving a genetic defect in transthyretin that is predominantly produced by the liver and amyloid is deposited in renal, gastrointestinal tract, myocardial, and nervous tissues. LT is a widely accepted treatment, and liver grafts from these patients can be used in recipients who are 55 years and older based on the concept that the latency period for the onset of symptoms related to amyloid deposition is approximately 20 years.

\section{Auxiliary partial orthotopic liver transplantation (APOLT)}

In this strategy, part or whole of the native liver is left in situ and part or whole of a donor liver is implanted in addition [43]. If the native liver recovers from the initial insult such as a viral hepatitis, then stopping the immunosuppression will cause atrophy of the implanted liver in due course, leaving the patient with his own fully functioning liver with no need to take any further immunosuppression. This strategy is also useful in selected disorders of deficiency such as Criggler - Najjar syndrome which requires only a small portion of normal liver to correct the clinical deficiency. However, in such patients stopping immunosuppression is not possible.

\section{Immunosuppression}

Immunosuppression following transplantation is the key to graft and patient survival. With the rapid advances in this fascinating field of transplant immunology, there is a constant search for the ideal immunosuppression which adequately limits the immunity to prevent rejection, and at the same time does not precipitate infection, long-term metabolic sequelae and chronic renal impairment. Immunosuppression is therefore more of a personalised medicine with patient tailored immunosuppression.

\section{Rejection and types}

Importantly, liver with the largest reservoir of innate immunity in the form of kupffer cells, is an immunotolerant organ [44] with regard to transplantation and is subject to less aggressive immunological attack than other organs. When there is an activated $\mathrm{T}$ cell response on the allograft antigens, then it is referred to as rejection.

- Hyper-acute rejection [45] is rare and is due to pre-sensitization to donor antigens. It occurs within minutes to hours after portal vein reconstruction intraoperatively and is mediated by complement fixation resulting in intravascular thrombosis.

- Acute cellular rejection [46] is more common, and is characterized by activated cytotoxic $\mathrm{T}$ cells leading to an immune 
response in the allograft. This is initiated by the presentation of donor HLA- antigens to host T cells within the graft which, via the secretion of interleukin (IL)-2, recruit activated $\mathrm{T}$ cells within the graft, resulting in allograft injury and dysfunction. Acute cellular rejection requiring treatment can occur in 40 - $60 \%$ of recipients, commonly within the first 3 months immediate post-transplant period.

- Chronic cellular rejection [47] develops in approximately 5\% grafts. It is associated with progressive ductopenia and fibrosis and develops secondary to poor compliance to drugs or inadequate immunosuppression, poor response to treatment. As a consequence there is poor graft and patient survival, most of the times necessitating re-transplantation.

Drugs for immunosuppression

Commonly used drugs are classified as corticosteroids, calcineurin inhibitors (CNI), anti-metabolites and inhibitors of TOR (target of rapamycin).

\begin{tabular}{|l|l|}
\hline \multicolumn{1}{|c|}{ Class } & \multicolumn{1}{c|}{ Drugs } \\
\hline General & Corticosteroids \\
\hline Calcineurin inhibitors & Tacrolimus, cyclosporine \\
\hline Antimetabolites & $\begin{array}{l}\text { Mycophenolate mofetil, mycophenolic } \\
\text { acid, azathioprine }\end{array}$ \\
\hline mTOR inhibitors & Sirolimus, everolimus \\
\hline Monoclonal antibodies & $\begin{array}{l}\text { OKT3, Basiliximab (IL-2), alemtuzumab } \\
\text { (anti CD 52) }\end{array}$ \\
\hline
\end{tabular}

Table 1

\section{Corticosteroids}

Corticosteroids are general anti-inflammatory agents with primary action on the inhibition of cytokine gene transcription. Hence, steroids prevent $\mathrm{T}$ cell recruitment and activation [48]. Long term steroid use can lead to hypertension, cushingoid facies, cataract, weight gain, dyslipidaemia, osteoporosis, post-prandial hyperglycaemia and diabetes.

\section{Calcineurin inhibitors (CNI)}

Upon recognition of donor antigens by the CD4 - T helper cells, there is IL-2 transcription by the calcineurin- calmodulin pathway. Consequently, an immune response is mounted on the allograft [49]. Hence, blocking of IL-2 production by inhibiting the calcineurin-calmodulin pathway, has made calcineurin inhibitors as the most important constituent of most of the immunosuppression protocols. Cyclosporine and tacrolimus are the two commonly used CNIs in our clinical practice. Dosage is monitored by measuring trough levels and maintaining them in the therapeutic range. Different centres have their experience on the levels to be maintained for adequate immunosuppression. In our own experience we found a tacrolimus concentration of 5 to $<8 \mathrm{ng} / \mathrm{mL}$ was associated with least overall toxicity, neurotoxicity, and incidence of rejection [50].
Side effects are common [51], while maintaining a high trough level for prolonged periods of time. The most significant side-effect of CNIs is nephrotoxicity, occurring in $40-70 \%$ patients, and occurs primarily as a consequence of intrarenal vasoconstriction. Renal impairment is the most common indication for dose reduction or cessation of treatment. Acute nephrotoxicity is reversible, whereas chronic toxicity is not; up to $5 \%$ may eventually require renal support; the onset of late renal failure is determined largely by the amount of exposure to CNI in the first year. Other frequent side effects include hypertension, venous thrombosis, tremor, headache, seizures, hyperkalaemia, gout and gingival hyperplasia.

\section{Antimetabolites}

Azathioprine, a prodrug form of 6-mercaptopurine, has a number of intracellular actions including inhibition of DNA synthesis via the purine metabolism and reduction in nucleotide synthesis. These actions lead to inhibition of T cell activation, and a decrease in the level of circulating granulocytes [52]. Azathioprine cannot be used on its own and always used in conjunction with steroids or CNIs. Significant side effects, include bone marrow suppression (especially in those who have low levels of thiopurine methyltransferase).

Mycophenolate [53] is a selective inhibitor of de novo purine synthesis and is a potent inhibitor of both $\mathrm{B}$ and $\mathrm{T}$ cell proliferation. Important side effects are gastrointestinal intolerance manifesting as loose stools, flatulence, and significantly bone marrow suppression. It is teratogenic in pregnant women.

\section{mTOR inhibitors}

Although structurally similar to tacrolimus, they are not CNIs and mainly act on the mTOR pathway leading to cell cycle arrest at the G1 to S phase [54], thereby reducing the proliferation of CD4 and CD8 T cells.

The principal side effects of sirolimus [55] include poor wound healing, hyperlipidaemia, thrombocytopaenia, anaemia, leucopenia and peripheral oedema. Sometime back, there was blackbox warning against the use of sirolimus due to reports showing its propensity to cause hepatic artery thrombosis.

Everolimus [56] is a congener of sirolimus, better tolerated and currently plays an important role in various CNI minimization protocols.

\section{Complications in LT}

LT carries a $30-40 \%$ risk of overall morbidity in the recipient [11]. Amongst the major complications are bleeding requiring reexploration (5\%); primary non-function (1-2\%); hepatic artery thrombosis (2-5\%); portal vein thrombosis (2\%); biliary complications including anastomotic leak and stricture (5-15\%); sepsis (bacterial, viral, fungal, mycobacterial); rejection (acute cellular 
or T cell mediated rejection; chronic rejection), renal dysfunction; and metabolic or systemic complications such as hypertension, diabetes, hyperlipidemia secondary to immunosuppressive drugs.

\section{Conclusion}

While we have discussed extensively, the basics of liver transplant in terms of indications, the types of LT and post-transplant immunosuppression, the bottom line is awareness amongst practising physicians as to when one he/or she should take an initiative to seek opinion or discuss with a liver specialist that is supported by a strong LT team and not lose the critical time when one can still salvage some of our patients. Timely referral is the key for a good outcome with reduced morbidity post-transplant. It is not uncommon to observe in our day to day practice patients being managed by primary care physicians, general practitioners and other specialities without referral to the transplant team, resulting in poor outcome due to failure to qualify even for registration for LT e.g. failure to identify complications like MHE, SBP or HCC.

\section{Disclosures}

None.

\section{Potential conflicts of Interest}

None.

\section{Funding}

None.

\section{Acknowledgements}

None.

\section{Bibliography}

1. Starzl TE., et al. "Homotransplantation of the liver in humans". Surgery, Gynecology, and Obstetrics 117 (1963): 659-676.

2. D'Amico G. "The clinical course of cirrhosis. Population based studies and the need of personalized medicine". Journal of Hepatology 60 (2014): 241-242.

3. D'Amico G., et al. "Natural history and prognostic indicators of survival in cirrhosis: a systematic review of 118 studies". Journal of Hepatology 44 (2006): 217-231.

4. Bernardi M., et al. "Mechanisms of decompensation and organ failure in cirrhosis: From peripheral arterial vasodilation to systemic inflammation hypothesis". Journal of Hepatology 63 (2015): 1272-1284.

5. Arvaniti V., et al. "Infections in patients with cirrhosis increase mortality four-fold and should be used in determining prognosis". Gastroenterology 139 (2010): 1246-1256.

6. Child GC II and Turcotte JG. "Surgery and portal hypertension". In: Child GC II, editor. The liver and portal hypertension. Philadelphia: Saunders (1964): 50-58.
7. Pugh RN., et al. "Transection of the oesophagus for bleeding esophageal varices". British Journal of Surgery 60 (1973): 646649.

8. Kamath PS., et al. "A model to predict survival in patients with end-stage liver disease". Hepatology 33 (2001): 464-470.

9. Malinchoc M., et al. "A model to predict poor survival in patients undergoing transjugular intrahepatic portosystemic shunts". Hepatology 31 (2000): 864-871.

10. Schrier RW., et al. "Peripheral arterial vasodilation hypothesis: a proposal for the initiation of renal sodium and water retention in cirrhosis". Hepatology 8 (1988): 1151-1157.

11. Merion RM., et al. "The survival benefit of liver trans-plantation". American Journal of Transplantation 5 (2005): 307-313.

12. Wiesner R., et al. "Model for end-stage liver disease (MELD) and allocation of donor livers". Gastroenterology 124 (2003): 91-96.

13. Freeman RB., et al. "Results of the first year of the new liver allocation plan". Liver transplantation 10 (2004): 7-15.

14. Angeli P., et al. "Hyponatremia in cirrhosis: results of a patient population survey". Hepatology 44 (2006): 1532-1542.

15. Londono MC., et al. "MELD score and serum sodium in the prediction of survival of patients with cirrhosis awaiting liver transplantation". Gut 56 (2007): 1283-1290.

16. Biggins SW., et al. "Evidence-based incorporation of serum sodium concentration into MELD". Gastroenterology 130 (2006): 1652-1660.

17. Kim WR and Biggins SW., et al. "Hyponatremia and mortality among patients on the liver transplant waiting list". The New England Journal of Medicine 359 (2008): 1018-1026.

18. Fallon MB., et al. "Model for end-stage liver disease (MELD) exception for hepatopulmonary syndrome". Liver Transplantation 12 (2006): S105-S107.

19. Krowka MJ., et al. "Model for end-stage liver disease (MELD) exception for porto-pulmonary hypertension". Liver Transplantation 12 (2006): S114-S116.

20. Mazzaferro V., et al. "Liver transplantation for the treatment of small hepatocellular carcinomas in patients with cirrhosis". The New England Journal of Medicine 334 (1996): 693-699.

21. Yao FY., et al. "Liver transplantation for hepatocellular carcinoma: expansion of the tumor size limits does not adversely impact survival". Hepatology 33 (2001): 1394-1403. 
22. Pomfret EA., et al. "Report of a national conference on liver allocation in patients with hepatocellular carcinoma in the United States". Liver Transplantation 16 (2010): 262-278.

23. Sasaki K., et al. "Development and validation of the HALT-HCC score to predict mortality in liver transplant recipients with hepatocellular carcinoma: a retrospective analysis". The Lancet Gastroenterology and Hepatology 2.8 (2017): 595-603.

24. The European Association for the Study of the Liver. "EASL Clinical Practice Guidelines for the management of patients with decompensated cirrhosis". Journal of Hepatology (2018).

25. Machiano VI. "Model for end stage liver disease-sodium score". Clinical Liver Disease 21 (2017): 275-287.

26. Bernal W., et al. "Acute liver failure". Lancet 376 (2010): 190201.

27. O'Grady JG., et al. "Acute liver failure: redefining the syndromes". Lancet 342 (1993): 273-275.

28. O'Grady JG., et al. "Early indicators of prognosis in fulminant hepatic failure". Gastroenterology 97 (1989): 439-445.

29. Sen S., et al. "The pathophysiological basis of acute-on-chronic liver failure". Liver 22.2 (2002): 5-13.

30. Sarin SK., et al. "Acute-on-chronic Liver Failure (ACLF): Consensus Recommendations of the Asian Pacific Association for the Study of the Liver (APASL)". Hepatology International 3 (2009): 269-282.

31. Moreau R., et al. "Acute-on-chronic liver failure is a distinct syndrome that develops in patients with acute decompensation of cirrhosis". Gastro 144 (2013): 1426-1437.

32. Choudhary A., et al. "Liver failure determines the outcome in patients with acute-on-chronic liver failure (ACLF): comparison of APASL ACLF research consortium (AARC) and CLIF-SOFA models". Hepatology International 11.5 (2017): 461-471.

33. Khanna R., et al. "Neonatal cholestasis with ductal paucity and steatosis". Indian Pediatrics 50 (2013): 316-320.

34. Deirdre Kelly. Diseases of the Liver and Biliary System in Children 2008.

35. Berg CL., et al. "Improvement in survival associated with adult-to-adult living donor liver transplantation". Gastroenterology 133 (2007): 1806-1813.

36. Merrill JP., et al. "Successful transplantation of kidney from a human cadaver". Journal of the American Medical Association 185 (1963): 347-353.
37. A definition of irreversible coma Report of the Ad Hoc Committee of the Harvard Medical School to Examine the Definition of Brain Death. Journal of the American Medical Association 205.6 (1968): 337-340.

38. Feng S., et al. "Characteristics associated with liver graft failure: the concept of a donor risk index". American Journal of Transplantation 6 (2006): 783-790.

39. Lopez-Navidad A and Caballero F. "Extended criteria for organ acceptance. Strategies for achieving organ safety and for increasing organ pool”. Clinical Transplantation 17 (2003): 308 324

40. Bismuth $\mathrm{H}$ and Houssin D. "Reduced-size orthotopic liver graft for liver transplantation in children”. Surgery 95 (1984): 367 370

41. Carrera MT., et al. "Domino liver transplantation: a practical option in the face of the organ shortage". Progress in Transplantation 13 (2003): 151-153.

42. Yamamoto S., et al. "Liver transplantation for familial amyloidotic polyneuropathy (FAP): a single center experience over 16 years". American Journal of Transplantation 7(11) (2007) 2597-2604

43. Rammohan A., et al. "Auxiliary partial orthotopic liver transplantation for selected noncirrhotic metabolic liver disease". Liver Transplantation 25.1 (2019): 111-118.

44. Vilatoba M., et al. "New immunosuppressive strategies in liver transplantation: balancing efficacy and toxicity". Current Opinion in Organ Transplantation 8 (2003): 139-145.

45. Wiesner RH., et al. "Acute hepatic allograft rejection: incidence, risk factors, and impact on outcome". Hepatology 28 (1998): 45.

46. Neuberger J and Adams DH. "What is the significance of acute liver allograft rejection?". Journal of Hepatology 29 (1998): 143-150.

47. Lowes JR., et al. "Chronic rejection of the liver allograft". Gastroenterology Clinics of North America 22 (1993): 401-420.

48. Mueller XM. "Drug immunosuppression therapy for adult heart transplantation. Part 1 . Immune response to allograft and mechanism of action of immunosuppressants". The Annals of Thoracic Surgery 77 (2004): 354-362.

49. Pfeilschifter J and Muhl H. "Immunopharmacology: anti-inflammatory therapy targeting transcription factors". European Journal of Pharmacology 375 (1999): 237-245. 
50. Varghese J., et al. "Tacrolimus related adverse effects in liver transplant recipients: its association with trough concentrations". Indian Journal of Gastroenterology 33.3 (2014): 219225.

51. Jiang $\mathrm{H}$ and Kobayashi M. "Differences between cyclosporin $\mathrm{A}$ and tacrolimus in organ transplantation". Transplantation Proceedings 31 (1999): 1978-1980.

52. Nielsen OH., et al. "The treatment of inflammatory bowel disease with 6-mercaptopurine or azathioprine". Alimentary Pharmacology and Therapeutics 15 (2001): 1699-708.

53. Moreno JM., et al. "Usefulness of mycophenolate mofetil in patients with chronic renal insufficiency after liver transplantation". Transplantation Proceedings 35 (2003): 715-717.

54. Koehl GE., et al. "Rapamycin protects allografts from rejection while simultaneously attacking tumors in immunosuppressed mice". Transplantation 77 (2004): 1319-1326.

55. Trotter JF. "Sirolimus in liver transplantation". Transplantation Proceedings 35 (2003): S193-200.

56. Tan HP., et al. "Everolimus: an update". Current Opinion in Organ Transplantation 8 (2003): 323-326.

\section{Assets from publication with us}

- Prompt Acknowledgement after receiving the article

- Thorough Double blinded peer review

- Rapid Publication

- Issue of Publication Certificate

- High visibility of your Published work

Website: www.actascientific.com

Submit Article: www.actascientific.com/submission.php

Email us: editor@actascientific.com

Contact us: +91 9182824667 